\title{
Three Baltic countries and Russian university students' attitudes toward foreigners
}

\author{
Kristi Kõiv, University of Tartu, Estonia
}

\section{Introduction}

The growth of immigration in Europe has been associated with an increase of antiforeigner attitudes in a variety of European countries (Gang, Rivera-Batiz, \& Yun, 2013). Immigrants (or foreigners) are perceived not only as outsiders in their new societies but also as a threat to the social, political and economic order as well as a threat to the cultural homogeneity and the national identity of the state (e.g. Scheepers, Gijberts, \& Coenders, 2002).

The growing body of research on attitudes toward out-group populations in general and foreigners in European countries reveal that most people express negative attitudes toward foreigners (Gorodzeisky \& Semyonov, 2009). Also, it was reported more prejudice toward foreigners perceived as culturally dissimilar as toward more similar foreigners (Asbrock et al., 2014).

The aim of the study examines similarities and differences between Estonian, Latvian Lithuanian and Russian university students' attitudes towards foreigners.

\section{Method}

Samples

Four samples of university students participated in the study: 118 Estonian (189 of them were women and 29 men), 101 Latvian (96 of them were women and 5 men), 101 Lithuanian (67 of them were women and 34 men), and 92 respondents (women 54 and 28 men).

\section{Instrument}

Questionnaire consists of four open-ended questions expressing subjective feelings of acceptance and un-acceptance of foreigners in four areas: (1) meaning of foreigners; (2) reasons for acceptance of foreigners; (3) reasons for non-acceptance of foreigners, and (3) influence of foreigners.

Quantitative content analysis was used to code and analyze the open-ended questions answers categories.

\section{Results}

Research results showed that four samples of students conceptualize foreigners mainly in society level: (1) differences in nationality and language, and (2) marginalization, whereby Russian students empathized more differences in nationality and language, and also differences in citizenship; and three Baltic counties students evaluated more differences in attitudes and values. Lithuanian university students conceptualize foreigners more often in terms of individual physical differences between people and Estonians expressed their own individualistic personal viewpoint by separating self from other groups of people

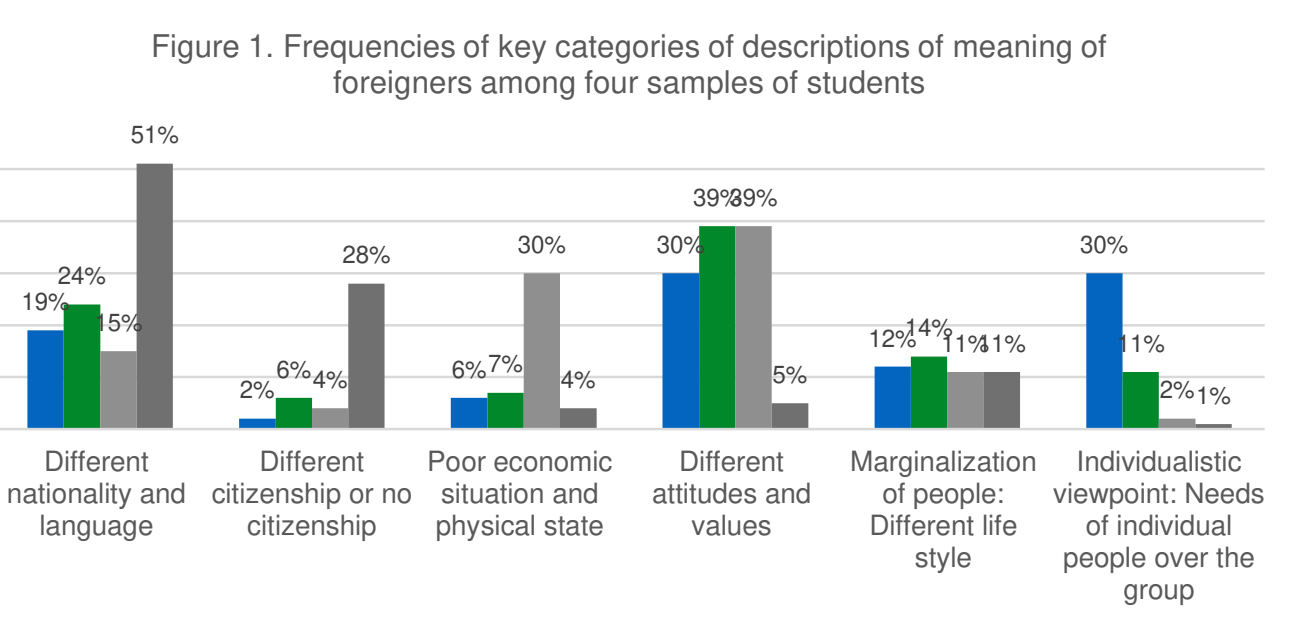

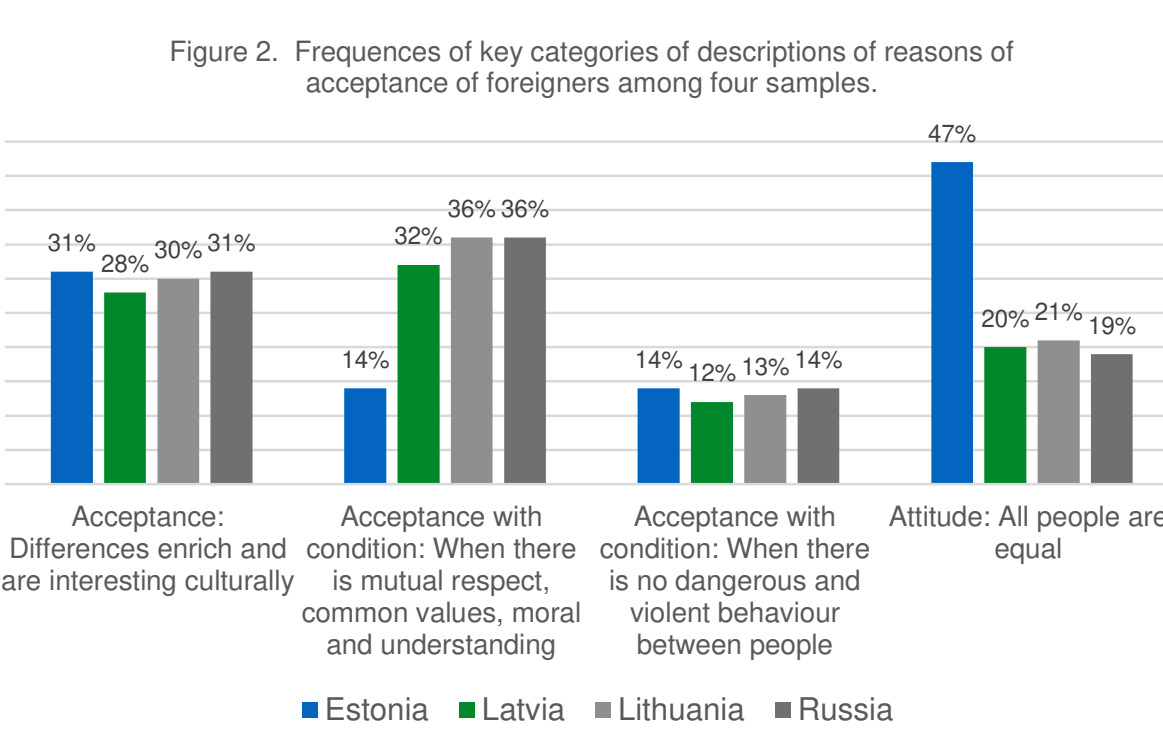

An analyze of reasons for acceptance of foreigners among university students reveal overwhelming positive attitude toward foreigners with regards to emplacing several cultures are enriching people. The reasons why young people in three Baltic countries accepted foreigners were different in personal level: (1) Estonian students were more prone to accept foreigners and otherwise minded people by expressing more often the attitude that all people are equal; (2) Latvian, Lithuanian and Russian students expressed more often the opinion to accept foreigners when there is a mutual respect, common values, moral and understandings between people.

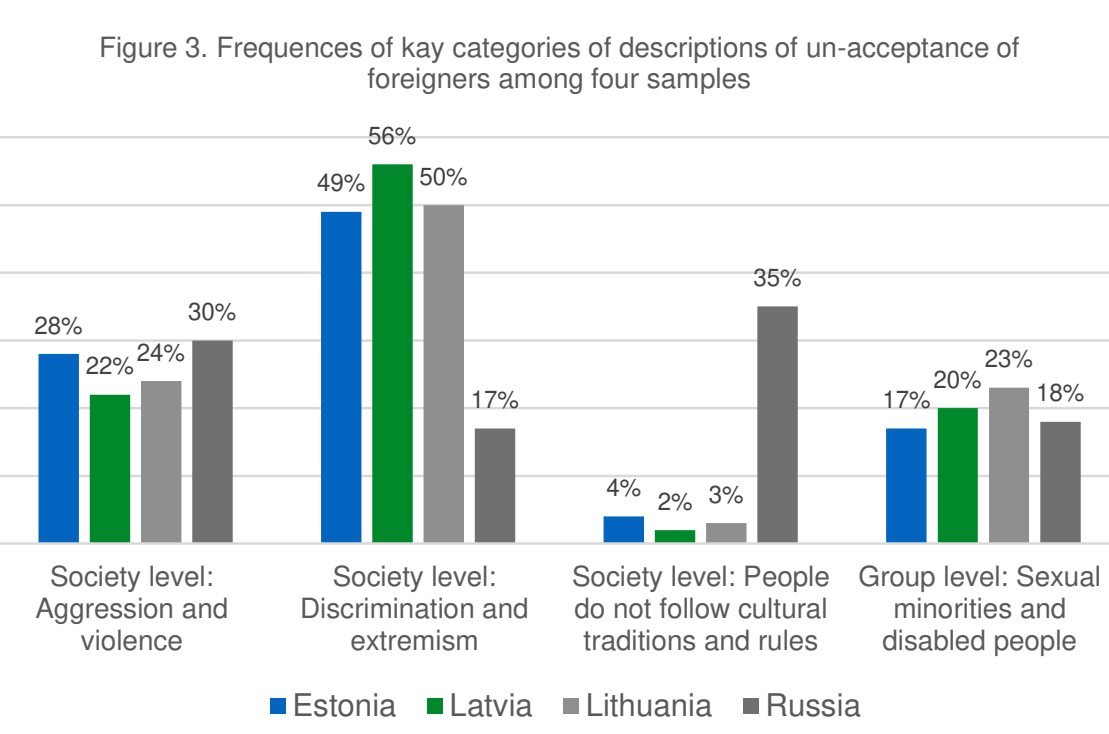

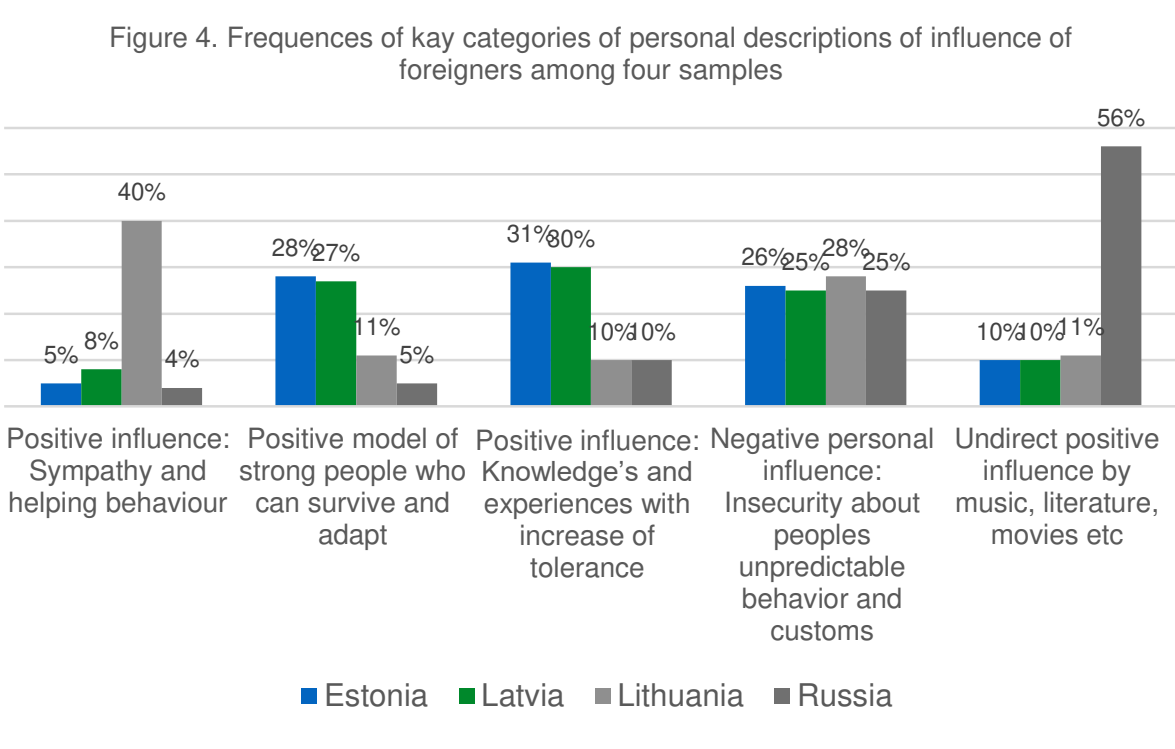

Conclusions

Following sociocultural perspectives present study reveal that university students understandings and interpretations of foreigners as socially contracted societal constructs differed somewhat - Russian respondents empathized more differences in citizenship and three Baltic states respondents in attitudes and values Additionally, it was revealed that the meaning of foreigners for Estonian students was conceptualized more from individualistic, than collectivistic, perspective.

The acceptance of foreigners of university students depends on the attitudes in the individual level: All four countries university students were generally open and tolerant toward foreigners in individual differences evoked from cultural enrichment, but their attitudes showed some variation: Estonian students expressed more the attitude that people are equal; and Latvian, Lithuanian and Russian respondents were more prone to express positive attitude towards foreigners when there is mutual respect and common values (Kõiv, Gurieva, Deyneka, Zuzeviciute, Liduma, \& Rone, 2019). 